DOI 10.37882/2223-2982.2020.12-2.28

\title{
АББРЕВИАЦИЯ КАК РАЗНОВИДНОСТЬ СУБСТИТУЦИИ АНГЛОЯЗЫЧНЫХ ТЕРМИНОВ СФЕР ГЕНЕТИКИ И ГЕННОЙ ИНЖЕНЕРИИ 1
}

\section{ABBREVIATION AS A VARIETY OF SUBSTITUTION \\ OF THE ENGLISH-LANGUAGE GENETICS AND GENETIC ENGINEERING TERMS ${ }^{2}$}

A. Razduyev

Summary: The article examines the phenomenon of substitution of the English-language genetic terms based on structural synonymy (doubling), in which a full-structure term in texts and discourse on the subject of Genetics and Genetic Engineering is replaced by an abbreviated unit. The purpose of the work is to identify the features of using the substitution of terms by specialists (and non-specialists) in special texts and discourse - replacing full-structure units with corresponding abbreviations of various types. In the course of the work, it has been found out that initial abbreviations (to a large extent with two and three components) are more often used during the substitution in comparison with syllabic abbreviations, truncations, contractions, acronyms, as well as blends, mixed and graphic abbreviations. The author comes to the conclusion about the significance of the substitution process in order to prevent repetitions of terminological units in texts and discourse and follow the principle of linguistic economy.

Keywords: abbreviation, substitution, term, Genetics, Genetic Engineering, discourse, text.
Раздуев Алексей Валерьевич К.филол.н., доцент, с.н.С., ФГБОУ ВО «Пятигорский государственный университет» arazduev@bk.ru

Аннотация: В рамках статьи изучается явление субституции англоязычных генетических терминов, базирующееся на структурной синонимии (дублетности), при котором полноструктурный термин в текстах и дискурсе по тематике генетики и генной инженерии заменяется на аббревиированную единицу. Целью работы является выявление особенностей применения субституции терминов специалистами (и неспециалистами) в специальных текстах и дискурсе - замен полноструктурных единиц на соответствующие аббревиатуры различных типов. В ходе работы обнаружено, что при субституции частотно используются инициальные аббревиатуры (в значительной степени ( двумя и тремя компонентами) по сравнению со слоговыми аббревиатурами, усечениями, стяжениями, акронимами, а также блендами, смешанными и графическими сокращениями. Автор приходит к выводу о значимости процесса субституции с целью недопущения повторов терминоединиц в текстах и дискурсе и следования принципу языковой экономии.

Ключевые слова: аббревиация, субституция, термин, генетика, генная инженерия, дискурс, текст.

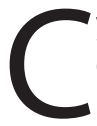
убституция представляет собой замещение одного объекта другим, обычно сходным по назначению или по функции; замену одной лексической единицы на другую. В рамках нашего исследования генетической терминологии под субституцией мы будем понимать замещение одного термина другим, обычно ему синонимичным (чаще дублетом, реже - вариантным соответствием). В частности, в статье рассматривается явление субституции, построенной на основе структурной синонимии (дублетности), при которой полноструктурный термин в текстах и дискурсе по тематике генетики и генной инженерии заменяется на аббревиированную единицу. Аббревиация анализируется нами как разновидность субституции англоязычных генетических терминов. Целью работы является выявление особенностей применения субституции терминов специалистами (и неспециалистами) в специальных текстах и дискурсе замен полноструктурных единиц на соответствующие аббревиатуры.

Методы исследования включают метод сплошной выборки англоязычных терминов сфер генетики и ген-

\footnotetext{
1 Исследование поддержано грантом Президента РФ (проект № 075-15-2019-347 «Деривационное, когнитивно-семантическое и дискурсивное моделирование русско-, англо- и испаноязычной терминологии сфер генетики и генной инженерии: источники возникновения терминов, история развития и современное состояние терминосистем»), ФГБОУ ВО «Пятигорский государственный университет», науч. рук. - канд. филол. наук, доц., старший науч. сотр. А.В. Раздуев.

2 The research was supported by the Russian Federation President grant (project 75-15-2019-347 «Derivative, cognitive-semantic and discursive modelling of the Russian, English and Spanish Genetics and Genetic Engineering terminology: the sources of terms, history of development and current state of the terminological systems»), Pyatigorsk State University, scientific advisor - A.V. Razduyev, PhD in Philology, Associate Professor, senior researcher.
} 
ной инженерии, а также текстовых и дискурсивных фрагментов, в которых данные термины употребляются, метод этимологического анализа, метод реконструкции структурно-словообразовательных моделей (моделей терминодеривации), методы компонентного и дефиниционного анализа для разбора структуры и семантики терминов, методы контекстуального, дискурсивного, дистрибутивного и контент-анализа, которые позволяют изучить особенности употребления терминологических единиц в текстах и дискурсе разных жанров.

Материалом для исследования послужила выборка англоязычных терминов сфер генетики и генной инженерии в количестве 2000 единиц, отобранных методом сплошной выборки из печатных и электронных источников по соответствующей тематике (из них по крайней мере 10\% могут иметь структурные дублеты-аббревиатуры (205 терминологических единиц)). Кроме того, были выбраны текстовые и дискурсивные фрагменты, в которых явление субституции генетических терминов проявляется наиболее ярко (не менее 500 фрагментов). Мы подчеркиваем тот факт, что аббревиация выступает достаточно частотным явлением в сфере генетики в связи с ее узкой специализацией, а относительно высокий процент аббревиированных единиц свидетельствует о недавнем возникновении анализируемых терминосистем (по сравнению с другими), их формирующемся характере, стремлении к краткости и схематичности, особенно на письме, желанию передать определенный объем информации в более короткий промежуток времени, затратив меньше усилий, уменьшить объем текста, при этом сохранив его смысловую нагрузку, особенно в том, что касается аннотаций научных статей, схем и рисунков, а также их описаний [см. также: Алимурадов, Шлепкина, 2010; Раздуев, 2015; Рудницкая, Алимурадов, 2019 и др.].

Среди частотно встречающихся типов аббревиатур в текстах и словарях по генетике и генной инженерии выделяются буквенные (инициальные) аббревиатуры, например, однокомпонентные (сокращения, состоящие из одной буквы) (37 единиц): adenosine -> A; absorbance $->A$; cytidine $->C$; deoxyribo ->d; guanosine -> G; restriction endonuclease -> R; methyltransferase -> M; nucleoside $->N$; operator $->0$, O; plasmid $->p$; promoter $->p$, $P$; resistance / resistant $->R$; restriction $->R$; (superscript) sensitivity/sensitive $->S$; sedimentation constant - $>S$; thymidine - $T$; terminator of transcription / transcription terminator $->t, T$; unit(s) - $>u$; uridine ->U; pyrimidine -> $Y$ [https://www.elsevier.com/_data/ promis_misc/gene_abbreviations.pdf; https://medicine. uiowa.edu/humangenetics/resources/glossary-terms-andabbreviations; http://www.informatics.jax.org/mgihome/ nomen/gene.shtml; https://www.multitran.com и др.] и др. Следует отметить тот факт, что некоторые однобуквенные аббревиатуры могут иметь несколько расшифровок даже в рамках генетической сферы, а также полную фор- му, состоящую из нескольких терминоэлементов (например, $t$ - terminator of transcription / transcription terminator, $R$ - restriction endonuclease и т.д.), при различиях в написании заглавными и строчными буквами - могут иметь разное значение.

Приведем некоторые примеры субституции полноструктурных терминологических единиц на аббревиатуры из научного дискурса:

Cytidine (C) to uridine (U) substitutional RNA editing is catalyzed by C4-cytidine deaminases that act on singlestranded RNA substrates (Figure 1B). C-to- $\boldsymbol{U}$ editing has been characterized in both plants and mammals [https://www.sciencedirect.com/topics/chemistry/cytidine].

Guanine, adenine, thymine, cytosine: G, A, T, C. These one-letter symbols have previously been established [1] and are generally used. There is, however, a problem of discriminating between the upper-case letters $\mathbf{G}$ and $\mathbf{C}$ on poorly copied sequences. Nevertheless, the use of alternative symbols for $\mathbf{G}$ (such as a barred-G) is not recommended. Discrimination between the lower-case letters is much clearer. Note that $T$ and $U$ may, in general, be considered as being synonyms, though care should be taken to avoid ambiguity in circumstances where it is likely. e.g. in discussing artificial hybrids of DNA and RNA and in cases where specific distinction between $\boldsymbol{T}$ and $\boldsymbol{U}$ is advisable [https://www.qmul.ac.uk/sbcs/iubmb/misc/naseq. html\#301]. В приведенных фрагментах произведена субституция генетических терминов, обозначающих азотистые основания, на однобуквенные аббревиатуры.

Далее рассмотрим двухкомпонентные аббревиированные единицы, встречающиеся в текстах по генетике (50 единиц), например: amino acid(s) $->$ aa, antibody(ies) $->A b$, base pair(s) -> bp, chloroplast $->c p$, dideoxyribo -> $d d$, double strand(ed) $->d s$, elongation factor $->E F$, heterozygous -> HT, homozygous $->H O$, initiation factor $->I F$, immunoglobulin(s) -> Ig, interleukin ->IL, insertion sequence(s) $->$ IS, kilobase(s) (= 1000 bp) $->$ kb, kanamycin $->$ Km, LuriaBertani (medium) -> LB, relative molecular mass (dimensionless) -> Mr, neomycin -> Nm, polyacrylamide -> PA, inorganic phosphate -> Pi, Shine-Dalgarno (sequence) -> SD, streptomycin $->S m$, single strand(ed) $->s s$, tetracycline $->T c$, thymidine kinase -> TK, ultraviolet -> UV, wild type -> wt [https://www. elsevier.com/_data/promis_misc/gene_abbreviations.pdf; https://medicine.uiowa.edu/humangenetics/resources/ glossary-terms-and-abbreviations; http://www.informatics. jax.org/mgihome/nomen/gene.shtml; https://www.multitran.com и др.] и др. Как видим, в данном случае большинство полноструктурных единиц являются двухкомпонентными, написание возможно как строчными, так и заглавными буквами.

Приведем в пример некоторые дискурсивные фрагменты, где наиболее ярко проявляется субституция терминологических единиц сфер генетики и генной инженерии. В частности, вместо полноструктурных единиц 
аббревиатуры широко употребляются на схемах и рисунках, в описаниях к ним, а также в аннотациях научных статей, где нужна краткость:

B6 mouse phenotyping Thirty days old wild-type (WT), heterozygous (HT) and homozygous (HO) B6 mice were sacrificed and evaluated for (A) gross appearance, (B) body weight (B6) $\left(n=3 ;{ }^{* *} p<0.02\right)$, (C) tooth eruption and (D) survival (B6). (E) X-ray analysis of hindlimbs, pelvis and vertebrae (upper panels) and histological examination of tibia proximal spongiosa (Masson's trichrome staining) (middle and lower panels). GP: growth plate. HBM: hematopoietic bone marrow. FBM: fibrotic bone marrow. T: trabecula. Middle panels; bar $=200 \mu \mathrm{m}$. Lower panels: bar $=30 \mu \mathrm{m}$. Histomorphometric evaluation of proximal tibia (F) trabecular bone volume/total tissue volume (BV/TV), (G) trabecular number (Tb.N), (H) trabecular thickness (Tb. Th) and (I) trabecular separation (Tb.Sp). Data are the mean \pm s.d. of 3 mice per group; ${ }^{*} p<0.05 ;{ }^{* *} p<0.002$ [https:// www.researchgate.net/figure/B6-mouse-phenotypingThirty-days-old-wild-type-WT-heterozygous-HT-andhomozygous_fig2_258253447].

Inorganic phosphate (Pi) is an essential nutrient to living organisms. It plays a key role in diverse biological processes, including osteoblast differentiation and skeletal mineralization. Maintenance of proper Pi homeostasis is a critical event, as any deviation from that state can lead to several acute and chronic disease states and influence the ageing process and lifespan [https://pubmed.ncbi.nlm.nih.gov/23394088/] и др. Следует отметить разную плотность употребления терминов и их аббревиированных субститутов в основных текстах научных статей (терминологическая плотность меньше) и рисунках и схемах, а также описанию к ним (больше).

Как видно из приведенных примеров, помимо однои двухкомпонентных аббревиатур в сферах генетики и генной инженерии наличествуют также трех- (46 единиц) и четырехкомпонентные (26 единиц) единицы. Приведем некоторые примеры из отобранных терминов: avian myeloblastosis virus -> AMV, bovine serum albumin -> $B S A$, gene encoding CAT -> cat, covalently closed circular -> ccc, Chinese hamster ovary -> CHO, counts per minute ->cpm, long terminal repeat(s) -> LTR, dithiothreitol -> DTT, human immunodeficiency virus -> HIV, Herpes simplex virus -> HSV, N6methyladenosine -> m6A, multiple cloning site(s) -> MCS, multiplicity of infection -> moi, nicotinamide-adenine dinucleotide ->NAD, open reading frame->ORF, polyethylene glycol->PEG, plaque-forming unit(s) -> pfu, inorganic pyrophosphate->PPi, ribosome-binding site(s) -> RBS, sodium dodecyl sulfate -> $S D S$, tobacco mosaic virus -> TMV, transcription start point(s) -> tsp, unidentified (open) reading frame -> URF, unstranslated region(s) -> UTR; restriction-fragment length polymorphism -> RFLP, cyclic adenosine 3',5'-monophosphate -> CAMP, DNA complementary to RNA -> CDNA, calf intestinal alkaline phosphatase -> CIAP, dimethylsulfoxide -> DMSO, deoxyribonucleoside triphosphate -> dNTP, high-performance liquid chromatography -> HPLC, hypoxanthine-guanine phosphoribosyl transferase -> HPRT, isopropyl $\beta$-D-thiogalactopyranoside -> IPTG, o-nitrophenyl $\beta$-D-galactopyranoside -> ONPG, phenylmethylsulfonyl fluoride -> PMSF, ribosomal RNA -> rRNA, DNA coding for rRNA -> rDNA [https://www.elsevier.com/_data/ promis_misc/gene_abbreviations.pdf; https://medicine. uiowa.edu/humangenetics/resources/glossary-terms-andabbreviations; http://www.informatics.jax.org/mgihome/ nomen/gene.shtml; https://www.multitran.com и др.] и др.

Как трех-, так и четырехкомпонентные аббревиатуры достаточно частотно встречаются в научных текстах и дискурсе, например:

Guanosine triphosphate (GTP) is used by the body to form the guanylic acid units in ribonucleic acids (RNA's) [https://www.britannica.com/science/guanine].

Guanosine is a nucleoside comprising guanine attached to a ribose (ribofuranose) ring via a $\beta$-N9-glycosidic bond. Guanosine can be phosphorylated to become GMP (guanosine monophosphate), CGMP (cyclic guanosine monophosphate), GDP (guanosine diphosphate) and GTP (guanosine triphosphate) which are factors in signal transduction pathways [https://pubchem.ncbi.nlm.nih.gov/compound/Guanosine] и др. Если в приведенном выше примере из научного дискурса на данную группу сначала приводятся полноструктурные единицы (guanosine triphosphate, ribonucleic acids), для которых в скобках приводятся аббревиированные субституты (GTP, RNA's), то во втором примере, наоборот, сначала приводятся аббревиатуры, а затем их расшифровка. И в том, и в другом тексте в дальнейшем используются аббревиированные единицы.

Гораздо менее частотными в плане применения в ходе субституции являются слоговые аббревиатуры, усечения и стяжения (23 единицы), например: adenovirus -> Ad, erythromycin -> Er, origin(s) of DNA replication -> ori, oligodeoxyribonucleotide -> oligo, $\beta$-galactosidase -> $\beta G a l$, hygromycin $->H y$, kilodalton(s) $->k D a$, recombinant $->$ re-, rifampicin $->$ Rif, thiostrepton $->$ Th; ampicillin $->$ Ap, Interferon -> IFN, chloramphenicol $->\mathrm{Cm}$, gentamicin $->\mathrm{Gm}$, mitochondria(I) $->m t$, nucleotide(s) $->n t$, transposon $->\mathrm{Tn}$ [https://www.elsevier.com/_data/promis_misc/gene_abbreviations.pdf; https://medicine.uiowa.edu/humangenetics/resources/glossary-terms-and-abbreviations; http:// www.informatics.jax.org/mgihome/nomen/gene.shtml; https://www.multitran.com и др.] и др. Следует также отметить преобладание в данной группе двух- и трехкомпонентных единиц.

Приведем примеры из научных и научно-популярных текстов и дискурса:

Interferons (IFNs) are a family of cytokines that were first identified almost half a century ago through their antiviral properties. IFNs not only have important antiviral effects but also have a role in antitumor and immunomodulatory responses. There are two major classes of IFNs: type I (IFN-a subtypes, 
IFN- $\beta$, etc.) and type II (IFN- $\gamma$ ) [https://www.sciencedirect. com/topics/biochemistry-genetics-and-molecular-biology/ interferon]. В данном примере термин Interferon(s) заменяется на аббревиатуру-стяжение INF(s).

Binding of zinc to a 19 mer double-stranded oligodeoxyribonucleotide was investigated by anodic stripping voltammetry and cyclic voltammetry in order to understand the roles of zinc in DNA cleavage catalyzed by mung bean nuclease. These methods rely on the direct monitoring of zinc oxidation current in the absence and in the presence of the oligo [https://ainfo.cnptia.embrapa.br/digital/bitstream/ item/177880/1/ID-24577.pdf] и др. В рамках приведенного дискурсивного фрагмента многокомпонентный термин oligodeoxyribonucleotide заменяется на усечение oligo.

Весьма интересными в плане создания и употребления в случае замены полноструктурных генетических терминов выступают акрофонетические аббревиатуры (акронимы) (12 единиц), например: S-adenosylmethionine -> AdoMet, S-adenosylmethionine -> SAM(e), Cm acetyltransferase -> CAT и др., некоторые из них представляют собой бэкронимы, т.е. составлены по уже существующему слову, например: enzyme-linked immunosorbent assay-> ELISA, PA-gel electrophoresis -> PAGE, 1,4-piperazinediethanesulfonic acid -> Pipes [https://www.elsevier.com/_data/ promis_misc/gene_abbreviations.pdf; https://medicine. uiowa.edu/humangenetics/resources/glossary-terms-andabbreviations; http://www.informatics.jax.org/mgihome/ nomen/gene.shtml; https://www.multitran.com и др.] и др. Приведем примеры субституции из научного дискурса:

S-Adenosyl-L-Methionine (SAMe): In Depth. What's the Bottom Line? How much do we know about SAMe? Research has evaluated several potential uses of SAMe, and a substantial number of studies on depression and liver diseases have been completed. However, some of the studies used injected SAMe, which may not have the same effects as SAMe taken as a dietary supplement [https://www.nccih.nih.gov/health/ sadenosyllmethionine-same-in-depth].

Analyses of deletion mutants of the gene for chloramphenicol (Cm) acetyltransferase (CAT) carried by the staphylococcal plasmid pUB112 revealed a regulatory region, which is indispensable for $\mathrm{Cm}$-inducible cat gene expression, located $70 \mathrm{bp}$ in front of the CAT-coding sequence [https://www.ncbi. nlm.nih.gov/pmc/articles/PMC554500/].

The enzyme-linked immunosorbent assay (ELISA) is an immunological assay commonly used to measure antibodies, antigens, proteins and glycoproteins in biological samples. Some examples include: diagnosis of HIV infection, pregnancy tests, and measurement of cytokines or soluble receptors in cell supernatant or serum. ELISA assays are generally carried out in 96 well plates, allowing multiple samples to be measured in a single experiment. These plates need to be special absorbant plates (e.g. NUNC Immuno plates) to ensure the antibody or antigen sticks to the surface. Each ELISA measures a specific antigen, and kits for a variety of antigens are widely avail- able [https://www.immunology.org/public-information/ bitesized-immunology/experimental-techniques/enzymelinked-immunosorbent-assay] и др. В целом, акронимы могут иметь более широкое применение, так как они сходны с обычными словами, однако явной тенденции к акронимизации в рамках терминологии генетики не наблюдается. В приведенных примерах полноструктурные единицы заменяются на акрофонетические аббревиатуры $(S A M(e), C A T, E L I S A)$.

Относительно небольшую группу составляют смешанные сокращения, бленды, графические и другие типы аббревиатур, которые используются для замещения полноструктурных терминов (всего 11 единиц): deoхуribonuclease -> DNase, restriction endonuclease -> ENase, DNA methyltransferase -> MTase, ribonuclease -> RNase, ethidium bromide -> EtdBr, Geneticin -> G(-)418, lac promoter-operator $->$ lacZpo, monoclonal Ab (antibody(ies)) -> $m A b$, million years -> Myr, 5-bromo-4-chloro-3-indolyl $\beta$-D-galactopyranoside -> Xgal, deletion $->\Delta$ [https://www.elsevier.com/_data/promis_misc/gene_abbreviations.pdf; https://medicine.uiowa. edu/humangenetics/resources/glossary-terms-and-abbreviations; http://www.informatics.jax.org/mgihome/nomen/ gene.shtml; https://www.multitran.com и др.]. Приведем некоторые примеры из текстов:

A schematic overview of the human canonical ribonucleases (RNases) in the host defence system. (A, B) RNases show antiviral activity and cytotoxic properties in mammalian cells and degrade RNA; (C) Cells of the immune system (immune cells, or ICs) secrete and are modulated by RNases; (D, F) Different mature cells secrete RNases, including endothelial (EnC) and epithelial (EpC) cells, for pericellular homeostasis; (E) Antimicrobial activity against bacteria, fungi and parasites has been demonstrated for RNases [https://www.ncbi.nlm.nih. gov/pmc/articles/PMC5000675/]. В данном фрагменте в виде субститутов употреблены как смешанные (ribonucleases -> RNases), так и инициальные аббревиатуры (immune cells $->I C$, endothelial cells $->$ EnC, epithelial cells $->$ $E p C)$.

G418, Geneticin. Commonly known as $\mathbf{G 4 1 8}$ or $\mathbf{G - 4 1 8 ,}$ Gibco Geneticin reagent is an aminoglycoside related to gentamicin, and is commonly used as a selective agent for eukaryotic cells. As an analog of neomycin sulfate, it interferes with the function of 805 ribosomes and protein synthesis in eukaryotic cells [https://www.thermofisher.com/ru/ru/home/life-science/cell-culture/transfection/selection/g418.html] и др. В рассматриваемом дискурсивном фрагменте употреблены полноструктурный термин Geneticin и креолизованные аббревиатуры G418 / G-418, представляющие собой номенклатурные наименования.

Таким образом, проанализировав выборку англоязычных терминов из сфер генетики и генной инженерии общим объемом 2000 единиц, мы выявили тот факт, что по крайней мере $10 \%$ из них могут иметь структурные дублеты-аббревиатуры (205 единиц), которые исполь- 
зуются в рамках процесса субституции. Отмечается частотная реализация субституции посредством использования инициальных аббревиатур (главным образом, с двумя и тремя компонентами, в меньшей степени с одним и четырьмя) по сравнению со слоговыми аббревиатурами, усечениями, стяжениями, акронимами, а также блендами, смешанными, графическими и прочими сокращениями. В целом, мы приходим к выводу о значимости процесса субституции генетических терминов с целью недопущения повторов одних и тех же единиц в научных текстах и дискурсе, а также следованию принципу языковой экономии.

\section{ЛИТЕРАТУРА}

1. Алимурадов 0.А., Шлепкина М.А. Инновационные лексические процессы в системе стереотипных конструкций современного англоязычного делового дискурса: аббревиация и сокращение // Филологические науки. Вопросы теории и практики. 2010. № 1-1 (5). С. $20-29$.

2. Мультитран. Электронный словарь. URL: https://www.multitran.com (дата обращения: 20.10.2020).

3. Раздуев А.В. Сокращение нанотехнологического термина как следование принципу речевой экономии в англоязычной профессиональной коммуникации // Когнитивные исследования языка. 2015. № 21. С. 518-522.

4. Рудницкая Д.Ю., Алимурадов 0.А. Аббревиация в современной английской терминосистеме сферы логистики: основные модели создания и структурные типы терминов-аббревиатур // Вестник Пятигорского государственного университета. 2019. № 2. С. 82-89.

5. Bruckner R., Matzura H. Regulation of the inducible chloramphenicol acetyltransferase gene of the Staphylococcus aureus plasmid pUB112. URL: https://www. ncbi.nlm.nih.gov/pmc/articles/PMC554500/ (дата обращения: 20.10.2020).

6. Charles E.S. RNA Substrates that Undergo C-to-U Editing. URL: https://www.sciencedirect.com/topics/chemistry/cytidine (дата 0бращения: 20.10.2020).

7. Enzyme-linked immunosorbent assay (ELISA). URL: https://www.immunology.org/public-information/bitesized-immunology/experimental-techniques/enzymelinked-immunosorbent-assay (дата обращения: 20.10.2020).

8. G418, Geneticin. URL: https://www.thermofisher.com/ru/ru/home/life-science/cell-culture/transfection/selection/g418.html (дата 0бращения: 20.10.2020).

9. Glossary of Terms and Abbreviations. lowa Institute of Human Genetics. URL: https://medicine.uiowa.edu/humangenetics/resources/glossary-terms-andabbreviations (дата обращения: 20.10.2020).

10. Guanine. Encyclopaedia Britannica. URL: https://www.britannica.com/science/guanine (дата обращения: 20.10.2020).

11. Guanosine. PubChem. National Library of Medicine. URL: https://pubchem.ncbi.nlm.nih.gov/compound/Guanosine (дата 0бращения: 20.10.2020).

12. Guidelines for Nomenclature of Genes, Genetic Markers, Alleles, and Mutations in Mouse and Rat. Mouse Genome Informatics. URL: http://www.informatics.jax. org/mgihome/nomen/gene.shtml (дата обращения: 20.10.2020).

13. Interferons. URL: https://www.sciencedirect.com/topics/biochemistry-genetics-and-molecular-biology/interferon (дата обращения: 20.10.2020).

14. Korzera P. et al. The Ribonuclease A Superfamily in Humans: Canonical RNases as the Buttress of Innate Immunity. URL: https://www.ncbi.nlm.nih.gov/pmc/ articles/PMC5000675/ (дата обращения: 20.10.2020).

15. List of abbreviations. URL: https://www.elsevier.com/_data/promis_misc/gene_abbreviations.pdf (дата обращения: 20.10.2020).

16. Moss G.P. Nomenclature for Incompletely Specified Bases in Nucleic Acid Sequences. URL: https://www.qmul.ac.uk/sbcs/iubmb/misc/naseq.html\#301 (дata 06 ращения: 20.10.2020).

17. Muraca M. Figure 2. B6 mouse phenotyping. URL: https://www.researchgate.net/figure/B6-mouse-phenotyping-Thirty-days-old-wild-type-WT-heterozygousHT-and-homozygous_fig2_258253447 (дата обращения: 20.10.2020).

18. Pires de Castro C.S. The binding of zinc (II) to a double-stranded oligodeoxyribonucleotide. URL: https://ainfo.cnptia.embrapa.br/digital/ bitstream/ item/177880/1/ID-24577.pdf (дата обращения: 20.10.2020).

19. S-Adenosyl-L-Methionine (SAMe): In Depth. URL: https://www.nccih.nih.gov/health/sadenosyllmethionine-same-in-depth (дата обращения: 20.10.2020).

20. Spina A. et al. Inorganic phosphate as a signaling molecule: a potential strategy in osteosarcoma treatment. URL: https://pubmed.ncbi.nlm.nih.gov/23394088/ (дата обращения: 20.10.2020).

(c) Раздуев Алексей Валерьевич (arazduev@bk.ru). 